\title{
Effects of low dose of aliskiren on isoproterenol-induced acute myocardial infarction in rats
}

\author{
I Bin-Jaliah", AM Hussein ${ }^{2}$, HF Sakr ${ }^{2,3}$, EA Eid ${ }^{4}$ \\ ${ }^{1}$ Department of Physiology, College of Medicine, King Khalid University, Abha, Saudi Arabia \\ ${ }^{2}$ Medical Physiology Department, Faculty of Medicine, Mansoura University, Mansoura, Egypt \\ ${ }^{3}$ Department of Physiology, College of Medicine and Health Sciences, Sultan Qaboos University, Muscat, Oman \\ ${ }^{4}$ Internal Medicine Department, Delta University for Science and Technology, Gamasa, Egypt
}

Received: June 23, 2017

Accepted: March 15, 2018

\begin{abstract}
This study examined the effects of aliskiren (Ali) (direct renin inhibitor) on serum cardiac enzymes (LDH and CK-MB), electrocardiography (ECG) changes, myocardial oxidative stress markers (MDA, CAT, and GSH) and the expression of $\mathrm{Bcl} 2$, HO-1, and Nrf2 genes in isoproterenol (ISO)-induced myocardial infarction (MI). A total of 40 male albino rats were allocated into four groups, (1) normal control (NC) group, (2) Ali group (rats received Ali at $10 \mathrm{mg} / \mathrm{kg} / \mathrm{day}$ p.o. for 5 days), (3) ISO group (rats received ISO $150 \mathrm{mg} / \mathrm{kg}$ i.p. for two consecutive days at $24 \mathrm{~h}$ intervals), and (4) Ali + ISO group (rats received ISO + Ali at $10 \mathrm{mg} / \mathrm{kg}$ /day p.o. for 5 days from the $2^{\text {nd }}$ dose of ISO). ISO group showed significant rise in serum cardiac enzymes (CK-MB and LDH), myocardial damage scores, myocardial MDA, HO-1, myocardial $\mathrm{Nrf} 2$ expression with significant reduction in myocardial antioxidants (CAT and GSH), and Bc12 expression compared to the normal group $(p<0.05)$. ECG showed ST segment elevation, prolonged QT interval and QRS complex, and increased heart rate in ISO group. Co-administration of Ali and ISO caused significant increase in cardiac enzymes and morphology with increase in MDA, serum K, and creatinine with significant decrease in Bcl2, HO-1, and Nrf2 without significant changes in ECG parameters compared to ISO group. We concluded that low dose of Ali seems to exacerbate the myocardial injury in ISO-MI, which might be due to the enhanced oxidative stress and apoptosis.
\end{abstract}

Keywords: aliskiren, isoproterenol, myocardial infarction, rats, $\mathrm{HO}-1, \mathrm{Nrf2}, \mathrm{Bcl} 2$

\section{Introduction}

World Health Organization (WHO) considers ischemic heart disease (IHD) as the main cause of morbidity and mortality in the world, which will be the major cause of death by the year 2020 (38). Myocardial infarction (MI), a type of IHD, is caused by imbalance between myocardial $\mathrm{O}_{2}$ supply and the demand that can be assessed by electrocardiography (ECG) $(26,48)$. Experimental MI can be induced by ligation of coronary arteries (ischemic MI) or by administration of $\beta$-adrenoceptor agonists, such as isoproterenol (ISO). ISO-induced MI is a well-established MI animal model that possesses the same pathophysiological mechanisms and morphological changes of human MI (14). Mechanisms underlying myocardial damage and cardiac arrhythmias in ISO-induced MI involve reactive oxygen species (ROS) and inflammatory cytokines production $(59,62)$. Recently, experimental studies have demonstrated a role for transcription factor Nuclear factor erythroid 2-related factor 2 (Nrf2), heme oxygenase-1 (HO-1), and apoptotic protein [such as B-cell/lymphoma 2 (Bcl2), Bax, and caspases-3 and -9] in ischemic and ISO-induced MI $(24,35,50)$.

Corresponding author: Abdel-Aziz M. Hussein, $\mathrm{PhD}$

Medical Physiology Department, Faculty of Medicine, Mansoura University, Mansoura 35516, Egypt

Phone: +20 100 2421140; Fax: +20 502 248203; E-mails: zizomenna28@yahoo.com; menhag@mans.edu.eg 
Renin-angiotensin system (RAS) plays an important role in the pathogenesis of many clinical conditions of the cardiovascular system, such as atherosclerosis, hypertension, MI, and heart failure (25). The RAS can be blocked at the levels of angiotensin (Ang)-converting enzyme (ACE) and Ang receptor (AR) and at the point of activation (direct renin inhibitors, DRI) (11). Aliskiren (Ali) is a non-peptide DRI that blocks the interaction between renin and angiotensinogen and prevents its cleavage to Ang I (55), leading to a decrease in all downstream components of the renin-angiotensin-aldosterone system (18). The role of RAS in the development of ischemic and ISO-induced heart failure still remains uncertain. Previous studies have demonstrated the benefits of the long-term use of RAS inhibitors including Ali in protection of myocardium after ischemic and ISO-induced injuries $(21,27,44,47,65,66)$. Also, Leenen et al. (33) demonstrated that the activity of circulatory RAS blunts the hypotensive effects of ISO-induced heart failure and treatment with ACEi and angiotensin receptor blockers (ARBs) did not prevent the ISO-induced cardiac hypertrophy. ARBs also enhanced the increase in myocardial collagen contact. Moreover, Guo et al. (19) demonstrated that ARBs failed to demonstrate any beneficial effect on ISO-induced myocardial hypertrophy and interstitial fibrosis. Thus, the effects of direct inhibition of RAS in early ISO-induced MI need more clarification. Therefore, this study was designed to investigate the effects of direct blockade of RAS using Ali on ECG changes, oxidative stress, and expression of Nrf2/HO-1 pathway and apoptotic markers in a rat model of acute ISO-MI.

\section{Materials and Methods}

\section{Experimental animals}

A total of 40 adult male Sprague Dawley rats aged 3-4 months and weighing between 150 and $180 \mathrm{~g}$ were maintained at room temperature at Nile Center for Experimental Research and had free access to food (standard pellet diet) and tap water ad libitum. This study was carried out according to the guidelines of experimental lab animal care and ethics and was approved by our local committee of ethics (approval no. R/16.12.87).

\section{Drugs and chemicals}

We purchased Tekturna tablets (Ali; $150 \mathrm{mg}$ tablets) from Novartis, America, and ISO hydrochloride from Sigma Chemical Co. (St. Louis, MO, USA).

\section{Experimental model of ISO-induced MI}

Animal model of ISO-induced MI was induced by i.p. administration of ISO $(150 \mathrm{mg} / \mathrm{kg}$ body weight; Sigma Chemical Co.) for two alternate days with 24-h intervals (52).

\section{Experimental design}

We randomly allocated rats into four groups as follows:

1. Normal control $(N C)$ group $(n=8)$ : normal rats received $0.5-\mathrm{ml}$ saline i.p. every alternate day for two alternate days and $0.5-\mathrm{ml}$ saline p.o. every day for 5 days.

2. Ali group $(n=8)$ : animals received Ali $(10 \mathrm{mg} / \mathrm{kg}$; (Rasilez tablets, Novartis Europharm Ltd. Co., UK; aliskiren $150 \mathrm{mg}$ ) p.o. for 5 days.

3. $I S O+M I$ group $(n=12)$ : animals received ISO only.

4. $($ Ali + ISO $)$ group $(n=12)$ : animals received ISO and Ali $(10 \mathrm{mg} / \mathrm{kg}$, orally for 2 days before the $1^{\text {st }}$ dose of ISO). 
Measurement of systolic BP and heart rate (HR)

Systolic BP and HR of animals were measured at basal (before ISO injection), and at Days 3 and 5 after the injection of ISO by a noninvasive rat tail BP monitor (ML 125 NIBP, AD Instruments, Australia) at the Medical Physiology Department, Mansoura Faculty of Medicine. The average of at least three measurements of BP was taken at each occasion.

ECG recording

In all rats, ECG recording was performed under light ether anesthesia at Day 0 (basal) and at Days 3 and 5 after induction of MI using MP36 Biopac data acquisition Student Lab System (software BSL 3.7.5, Medical Physiology Department, Mansoura Faculty of Medicine, Egypt). Lead II was recorded and the following ECG variables were measured: HR, QT intervals, QRS complex, and ST segment.

\section{Blood sample collection and harvesting of the heart}

We collected blood samples from the ophthalmic venous plexus of the rats by fine-walled Pasteur pipette under light halothane anesthesia. The collected blood was centrifuged and serum was stored at $-20^{\circ} \mathrm{C}$ until the time of assay of cardiac enzymes [lactate dehydrogenase (LDH) and creatine kinase-muscle/brain (CK-MB)]. In addition, at the end of the experiment, rats were sacrificed by high dose of $\mathrm{Na}^{+}$thiopental $(120 \mathrm{mg} / \mathrm{kg})$, then we opened the thorax and the heart was rapidly excised. The excised heart was washed in ice-cold isotonic saline and dissected into two parts; one part was stored in liquid nitrogen $\left(-170{ }^{\circ} \mathrm{C}\right)$ for PCR assay and biochemical analysis of oxidative stress markers, whereas the other part of the heart was stored in formalin $(10 \%)$ for histopathological examination.

Measurement of cardiac enzymes (CK-MB and LDH) and plasma renin activity (PRA) We measured the serum cardiac enzymes (LDH and CK-MB) with commercially available kits, according to the manufacturer's instructions (Bayer Diagnostics Ltd., Baroda, India and bioMérieux Diagnostics, Milan, Italy, respectively). PRA was determined by ELISA kit (cat no.: CAN-RA-4600) according to the manufacturer's instructions using Segma 690 microplate reader and the results were expressed as nanograms of Ang I/ml/h.

Estimation of oxidative stress markers [malondialdehyde (MDA), catalase (CAT), and glutathione (GSH)]

We homogenized a small part of cardiac tissue $(\sim 0.15 \mathrm{~g})$ in cold phosphate buffer saline $(\mathrm{pH}$ $7.4,50 \mathrm{mM})$, then aliquoted into different ependorffs and stored at $-20^{\circ} \mathrm{C}$. We assessed the concentration of MDA and reduced GSH and CAT activity in heart homogenates by colorimetric kits (Bio-Diagnostics, Giza, Egypt) according to the manufacturer's instructions.

Detection of mRNA of Nrf2, Bcl2, and HO-1 by RT-PCR in cardiac tissues

All procedures of RNA extraction and cDNA synthesis were done according to techniques previously described (23). Primers of the investigated genes were as follows: $\mathrm{Bcl} 2$, forward: atcgctctgtggatgactgagtac, reverse: agagacagccaggagaaacaac; glyceraldehyde 3-phosphate dehydrogenase, forward: ccagggctgcettctcttgt, reverse: ctgtgccgttgaacttgccg; HO-1, forward: tcaccttccegagcatcgac, reverse: tcaccctgtgcttgacctcg; Nrf2 (erythroidderived 2)-like 2 (Nrf2), forward: catttgtagatgaccatgagtcgc, reverse: gctccatgtcctgctgtatgc. About $10 \mu \mathrm{l}$ of PCR product was loaded on $2 \%$ agarose gel with ethidium bromide staining 
and the product was visualized on ultraviolet transilluminator and PCR products were semi-quantified by a gel documentation system (BioDocAnalyze, Nile Center for Experimental Research, Mansoura, Egypt).

\section{Histopathological examination}

The formalin-fixed cardiac tissues were processed into paraffin blocks and hemotoxylin and eosin staining was done. Cardiac tissues were examined for interstitial edema, inflammatory cells, and necrosis of myocardial cells. Briefly, the findings were graded as no changes; +mild (focal myocyte damage or small multifocal degeneration with slight degree of inflammatory process); ++moderate (extensive myofibrillar degeneration and/or diffuse inflammatory process); and +++ marked (necrosis with diffuse inflammatory process). According to the observed histopathological changes in myocardial tissue, all rats were divided into three groups: group A (no histopathological change), group B (mild histopathological change), and group C (moderate and/or severe histopathological changes) (41).

\section{Statistical analysis}

We measured the statistical significance among groups by using one-way analysis of variance test followed by Tukey's post hoc test. SPSS version 20 was used to perform the statistical analysis. $p$ value of $<0.05$ was considered significant.

\section{Results}

Results of animal survival in different groups

Kaplan curve showed that the survival rate was lesser in the Ali + ISO group compared to the $\mathrm{NC}$, Ali, and ISO groups. By the end of the experiment, 8 of 8 rats survived in the NC group, 10 of 12 rats in the Ali group, 9 of 12 rats in the ISO group, and 6 of 12 rats in the Ali + ISO group (Fig. 1).

Effects of Ali on biochemical markers (serum LDH, CK-MB, creatinine, $K^{+}$, and PRA) in ISO-MI

Basal values of serum cardiac enzymes (LDH and CK-MB), serum $\mathrm{K}$ and creatinine and PRA showed no statistical significance among all groups (NC, Ali, ISO, and Ali + ISO groups). In addition, compared to the NC group, the Ali group showed no statistically significant difference in these parameters at Days 3 and 5. At Days 3 and 5, the levels of LDH and CK-MB and PRA showed significant increase in the ISO and the Ali + ISO groups compared to the NC and the Ali groups $(p \leq 0.01)$. Furthermore, serum levels of CK-MB and LDH showed significant increase in the Ali + ISO group at Days 3 and 5 compared to the ISO and the Ali groups $(p<0.05)$, whereas PRA activity did not show any statistical significant difference between the ISO and the Ali + ISO groups. Regarding serum K and creatinine, they were significantly higher in the Ali + ISO group compared to the NC, Ali, and ISO groups at Days 3 and $5(p<0.05)$ (Table I).

Effect of Ali on body weight, heart weight/body weight, systolic BP, HR, and ECG variables (QT interval, QRS complex, T wave, and ST segment) in ISO-MI

At basal levels, body weight, heart weight/body weight, arterial blood pressure (ABP), HR, and ECG variables (QT interval, QRS complex, and ST segment) showed no statistically 


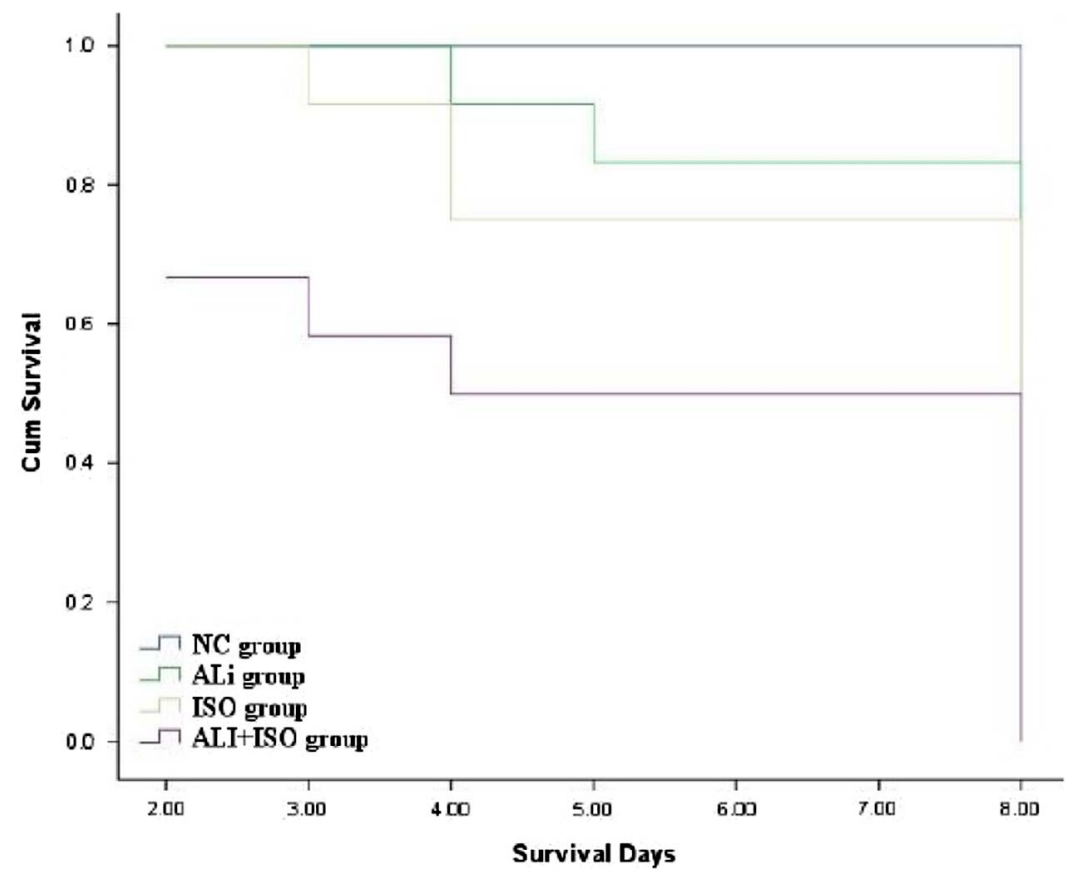

Fig. 1. Kaplan-Meier survival curves of the NC, Ali, ISO, and Ali + ISO groups

significant difference among all studied groups (NC, ISO, and Ali groups). There is no statistically significant difference between the Ali and the NC groups in all these parameters. Body weight was significantly lower and the heart weight/body weight ratio was higher in the ISO and the Ali + ISO groups compared to the NC and the Ali groups $(p<0.05)$ with no statistically significant difference between the ISO and the Ali + ISO groups at Days 3 and 5 . Systolic BP was significantly lower in the ISO and the Ali + ISO groups compared to the NC group $(p<0.05)$ with no statistically significant difference between the ISO and the Ali + ISO groups at Day 3, but it was significantly lower in the Ali + ISO group than in the ISO at Day $5(p<0.05)$. HR and ST segment, QT interval, and QRS complex were significantly higher in the ISO and the Ali + ISO groups than in the NC and the Ali groups at Days 3 and $5(p<0.01)$. QT interval, QRS complex, and ST segment showed no statistically significant difference between the ISO and the Ali + ISO groups (Table II). Figure 2a-d shows representative samples of ECG recordings at Day 0 from the NC, Ali, ISO, and Ali + ISO groups, respectively and Fig. 2e-h shows representative samples at Day 5 from the $\mathrm{NC}$, Ali, ISO, and Ali + ISO groups, respectively. ECG tracings showed increased T-wave amplitude in the ISO + Ali group (Fig. 2h).

Effect Ali on myocardial oxidative stress markers (MDA, CAT, and GSH) in ISO-MI Compared to the $\mathrm{NC}$ and the Ali groups, MDA concentration was significantly higher in the ISO and the Ali + ISO groups, while CAT activity and GSH concentration were significantly lower in the ISO and the Ali + ISO groups $(p<0.01)$. On the other hand, the Ali + ISO group showed a significant increase in MDA with significant decrease in GSH and CAT activity compared to the ISO-induced MI group ( $p<0.01$; Fig. 3a-c). 


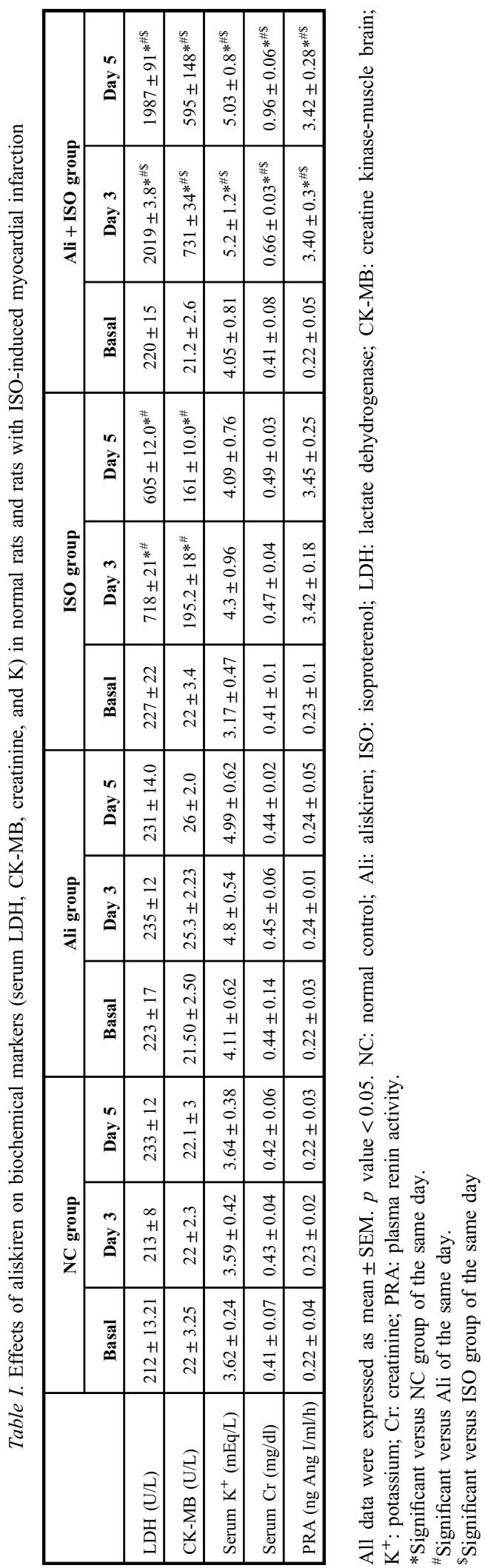




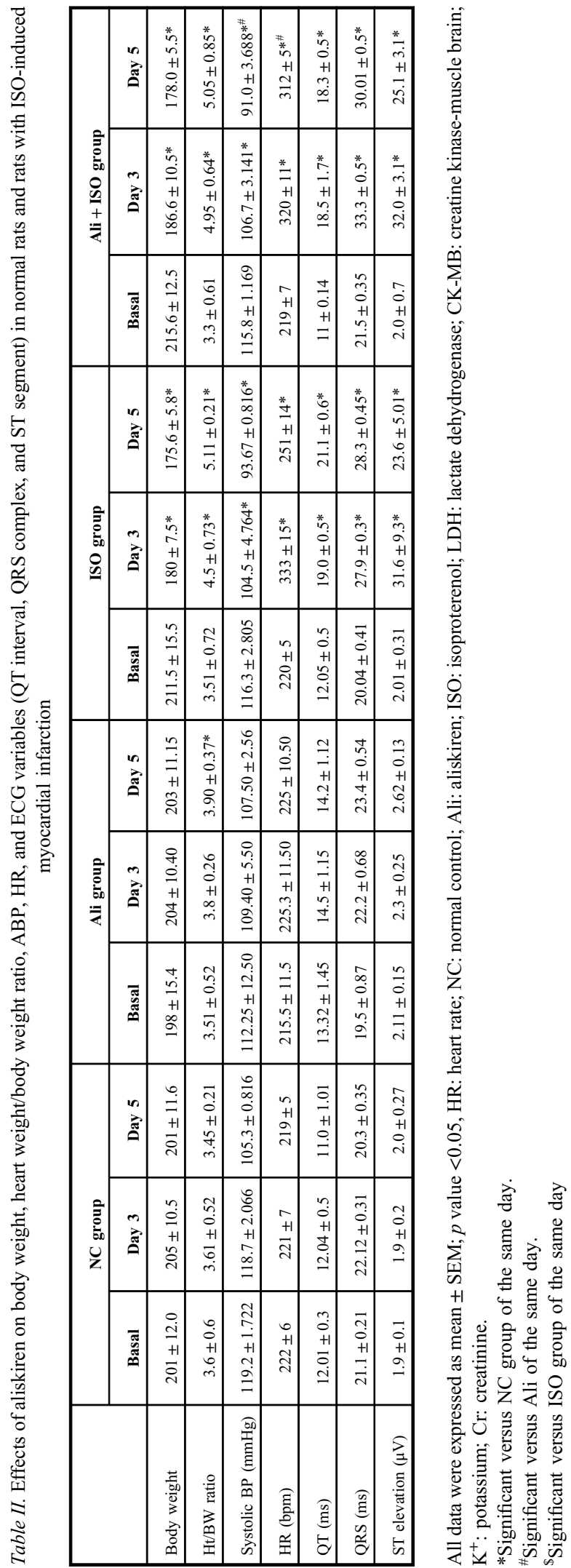



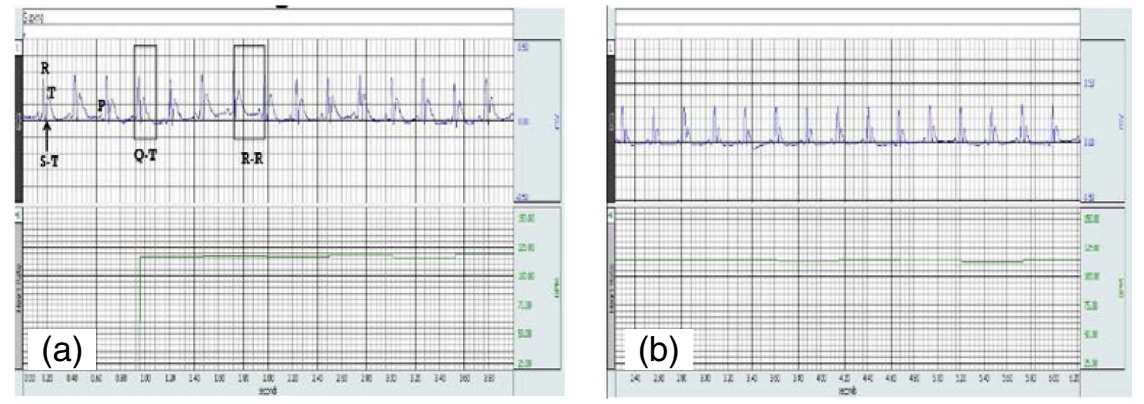

(b)
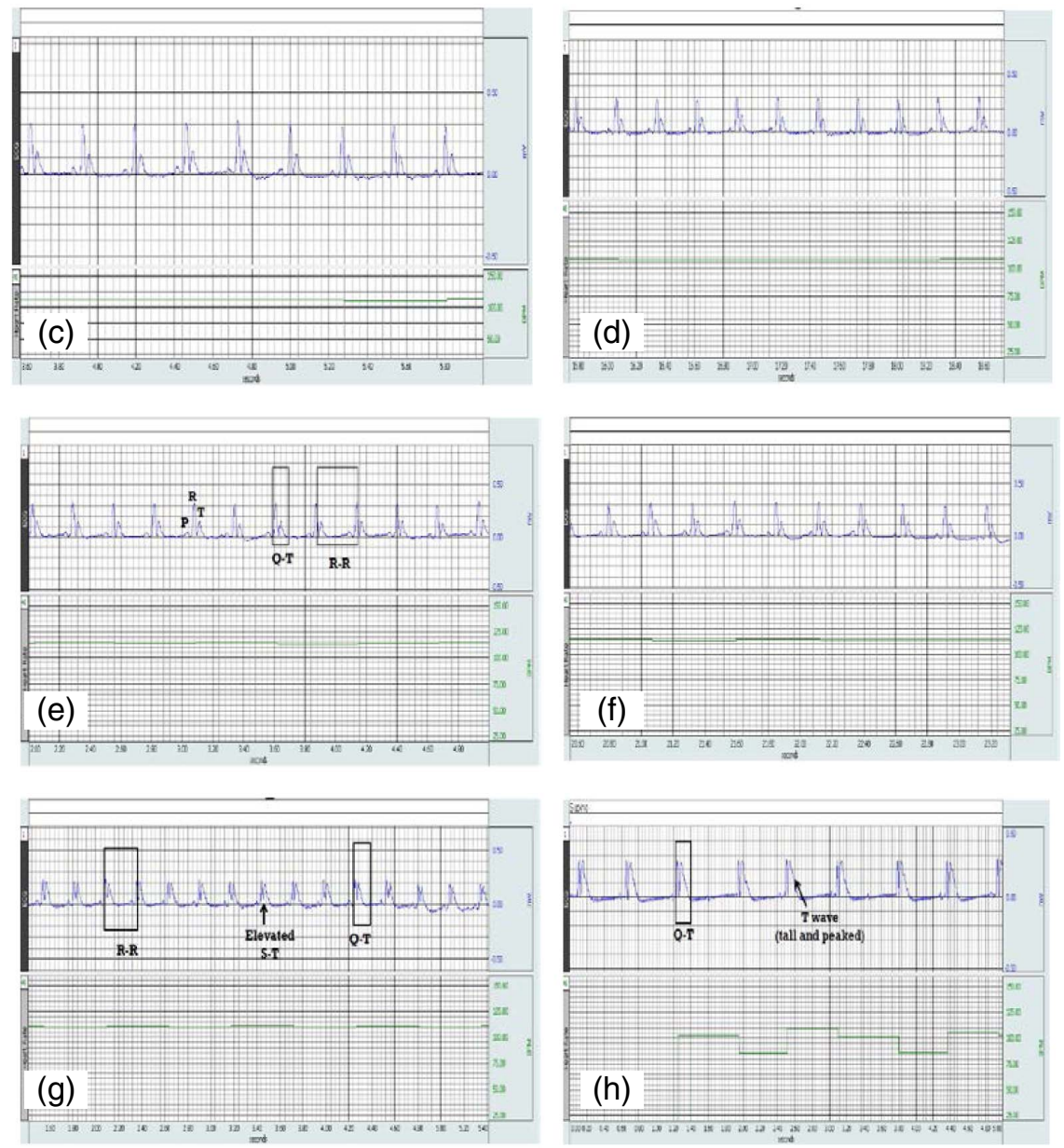

Fig. 2. Normal ECG waves and intervals in the NC group (a), the Ali group (b), the ISO group (c), and the Ali + ISO group (d) at Day 0 and in the NC group (e) at Day 5, tall peaked T-wave in the Ali group (f), elevated ST segment in the ISO group (g), and tall peaked T wave and elevated ST segment in the Ali + ISO group (h) at Day 5

Effect of Ali on myocardial expression of HO-1, Bcl2, and Nrf2 in ISO-MI Expression of mRNA of HO-1 in myocardium did not show statistically significant difference between the NC and the Ali groups, but it showed a significant increase in the ISO group 


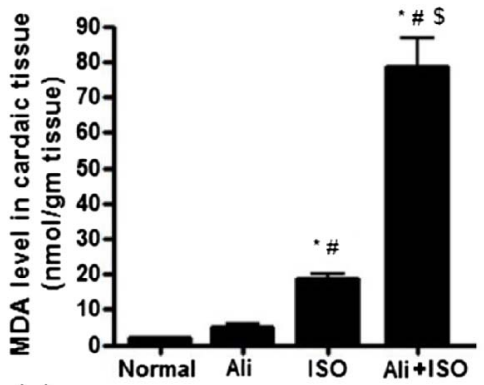

(a)

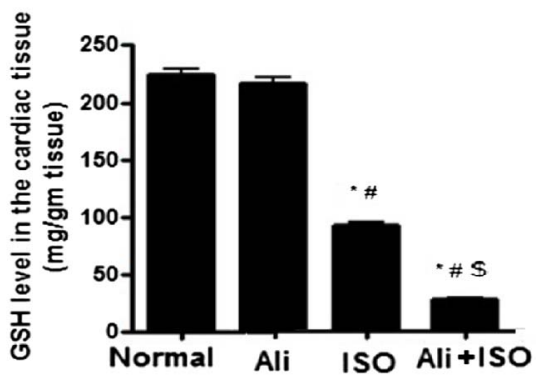

(b)

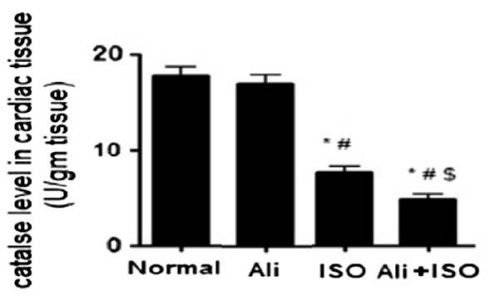

(c)

Fig. 3. Markers of oxidative stress in myocardial muscles. Concentrations of MDA (nmol/g heart tissues) (a), GSH (mg/g heart tissues) (b), and catalase activity (U/g heart tissues) (c) at Day 5. The symbol "*” denotes significant versus NC group, “\#” denotes significant versus Ali group, and “\$” denotes significant versus ISO group

compared to the $\mathrm{NC}$ and the Ali groups, and a significant decrease in the Ali + ISO group compared to the $\mathrm{NC}$ and the ISO groups $(p<0.01)$. On the other hand, Bcl2 expression in the myocardium showed a significant decrease in the ISO and the Ali + ISO groups compared to the $\mathrm{NC}$ and the Ali groups, and a significant decrease in the Ali + ISO group compared to the ISO groups $(p<0.01)$. Accordingly, Nrf2 expression in the myocardium showed a significant increase in the ISO group compared to the $\mathrm{NC}$ and the Ali groups, and a significant decrease in the Ali + ISO group compared to the NC and the ISO groups ( $p<0.01$; Fig. 4a-c).

\section{Effect of Ali on myocardial morphology in ISO-MI}

The score of myocardial damage in all rats from the $\mathrm{NC}$ group and most of rats in the Ali group was A, while most rats in the ISO and the Ali + ISO groups obtained score C (Table III). Figure 5a-d shows normal structure of myocardium from the NC group (Fig. 5a and b) and the Ali group (Fig. 5c and d) and Fig. 5e-g shows an infarcted zone with interstitial edema with massive inflammatory cells infiltration (ISO group) and myofibrillary degeneration. The hearts obtained from the Ali + ISO-treated rats showed massive myocardial necrosis with interstitial hemorrhage and neutrophil cell infiltration (Fig. 5h-j).

\section{Discussion}

The following are the main findings of this study. (1) ISO administration at a dose of $150 \mathrm{mg}$ caused significant deterioration in ECG parameters, elevation in oxidative stress, and apoptotic markers, and upregulation of HO-1 and Nrf2 expression. (2) Direct inhibition of RAS by low dose of Ali before ISO induction caused more significant deterioration in the 


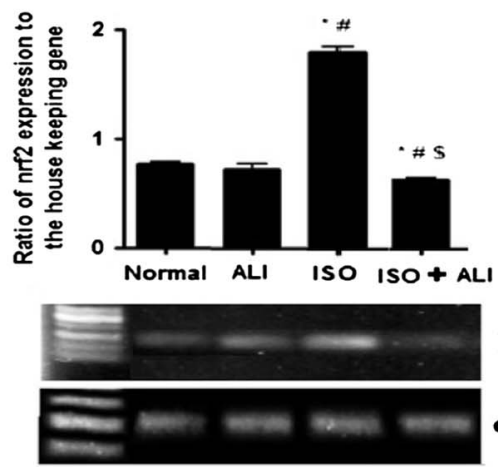

(a)

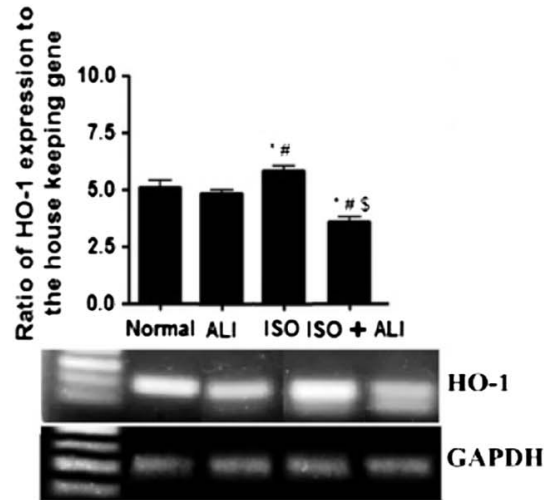

(b)

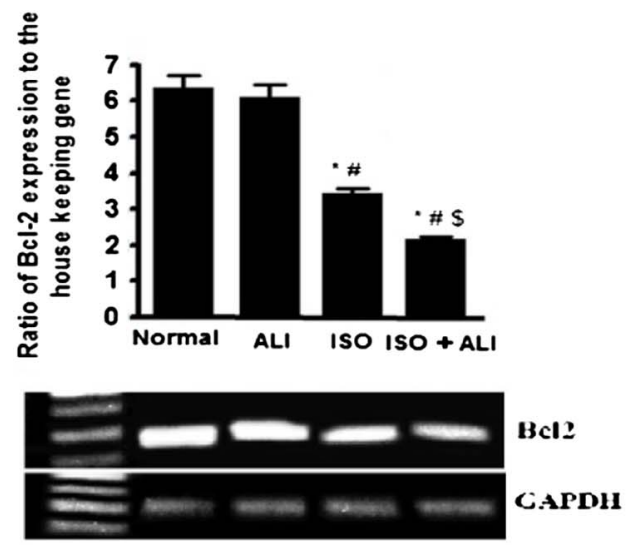

(c)

Fig. 4. The expression and RT-PCR products of Nrf2 (a), HO-1 (b), and Bcl2 (c) in myocardial muscles at Day 5. The symbol “*” denotes significant versus NC group, “\#” denotes significant versus Ali group, and “\$” denotes significant versus ISO group

Table III. Number of rats in each histopathological damage score of myocardium in different groups

\begin{tabular}{|l|c|c|c|}
\hline & Group A & Group B & Group C \\
\hline NC group & 8 & - & - \\
\hline Ali group & 8 & 2 & - \\
\hline ISO group & - & 4 & 5 \\
\hline Ali + ISO group & - & 2 & 4 \\
\hline
\end{tabular}

NC: normal control; ISO: isoproterenol; Ali: aliskiren; Group A: no histopathological changes; Group B: mild histopathological changes; Group C: moderate and/or severe histopathological changes 
studied parameters, whereas inhibition of RAS in normal rats did not cause any significant change in the studied parameters. In this study, we confirmed previous findings that showed a significant rise in serum levels of LDH and CK-MB in the ISO group, which was expected as a consequence of destruction of myocardial cell membranes by ISO $(12,41,46)$. In
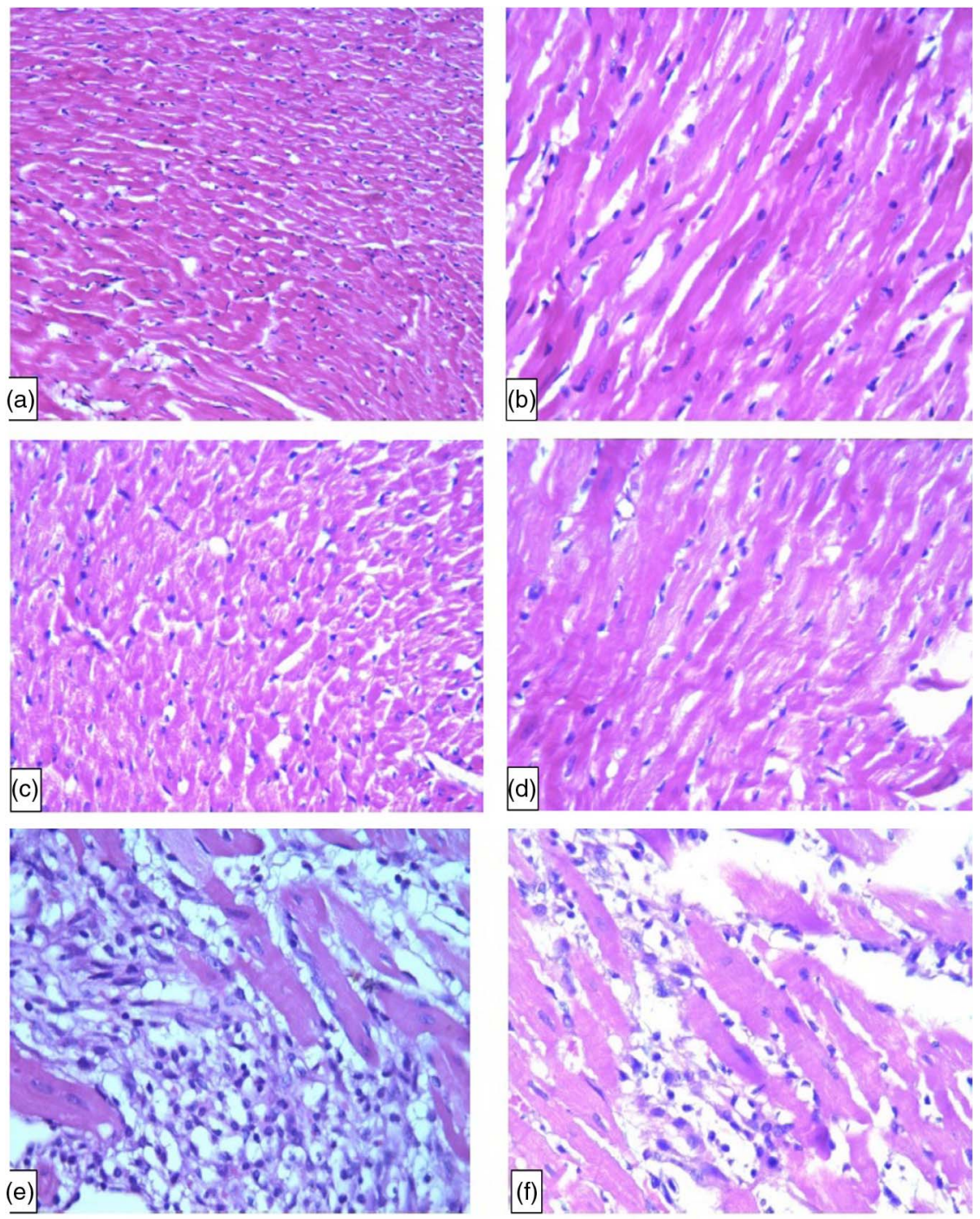

Fig. 5. Specimens of myocardium from the NC group showing normal architecture of rat myocardium at $200 \times$ (a) and $400 \times$ (b), from the Ali group showing normal architecture of rat myocardium at $200 \times$ (c) and $400 \times$ (d), from the ISO group, area of infarction with interstitial edema with marked neutrophil infiltration at $400 \times(\mathrm{e}-\mathrm{g})$, and from the Ali + ISO group, area of infarction with marked interstitial edema with acute extensive myofibrillary degeneration and hemorrhage (h), severe interstitial edema with marked neutrophil infiltration and moderate myofibrillary degeneration (i), and interstitial edema with interstitial hemorrhage (j) (H\&E, 400×) 

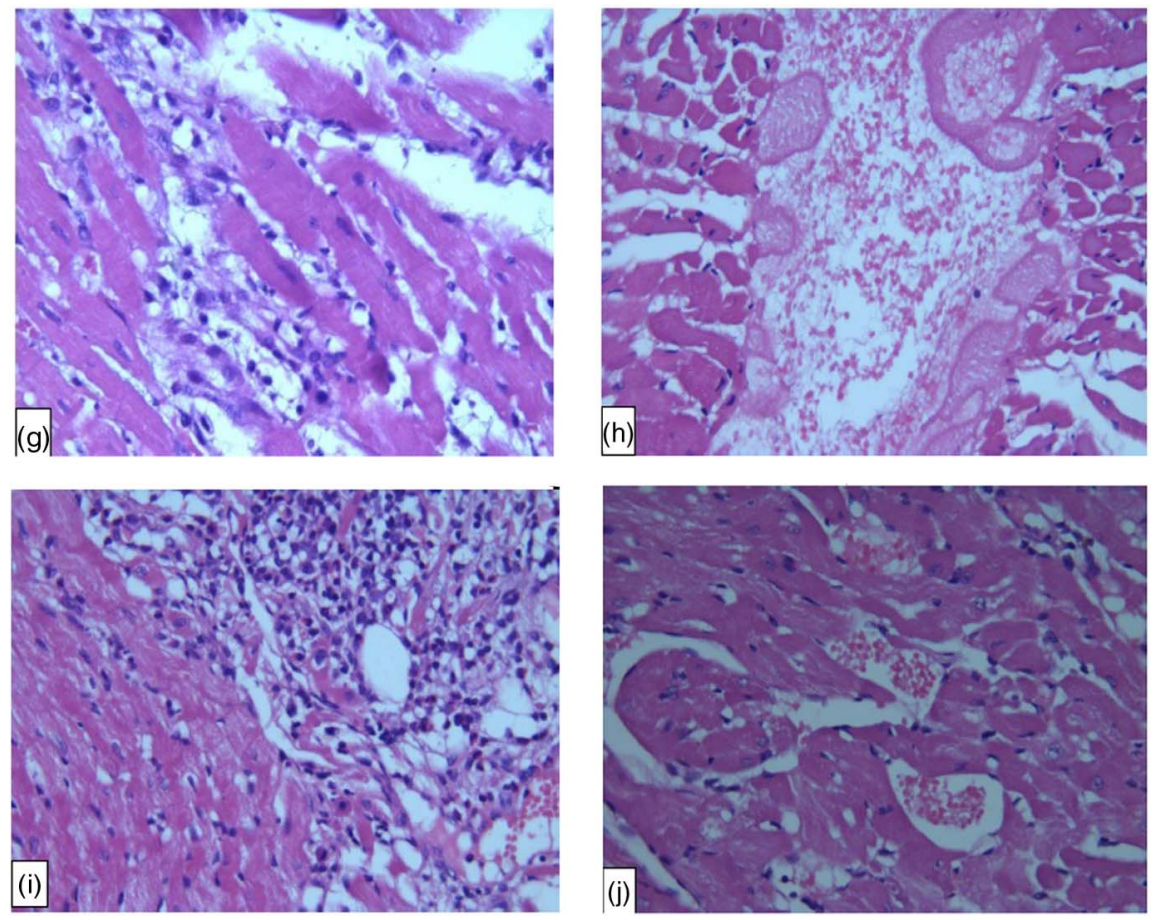

Fig. 5. (Continued)

consistence with functional abnormalities of cardiac functions, histopathological examination of cardiac tissues obtained from the ISO-MI group showed the characteristic findings of MI. These findings are similar to those reported by Dhalla et al. (12) and Metias et al. (41). Recently, Khan et al. (30) reported that administration of ISO $(85 \mathrm{mg} / \mathrm{kg})$ in two doses with 24-h intervals in rats caused a significant increase in markers of cardiac injury (LDH and CK-MB) with significant reduction in ABP, maximum rate of development of left ventricular (LV) pressure, and increase in LV end diastolic pressure.

Unfortunately, direct inhibition of RAS by low dose $(10 \mathrm{mg} / \mathrm{kg})$ of Ali before ISO-MI caused more significant deteriorations in the serum levels of cardiac enzymes (LDH and CK-MB) and myocardial histopathology compared to the ISO group, suggesting a negative role for RAS inhibition in early stages of ISO-MI. In agreement with these findings, Guo et al. (19) demonstrated that the inhibition of RAS with ARBs (Telm $10 \mathrm{mg} / \mathrm{kg}$ ) had no effect on ISO-induced myocardial hypertrophy and interstitial fibrosis. Also, Leenen et al. (33) demonstrated that the activity of circulatory RAS blunts the hypotensive effects of ISO-induced heart failure and treatment with ACEi (quinapril) and ARBs (losartan) did not prevent the ISO-induced cardiac hypertrophy as well as ARBs enhanced the increase in myocardial collagen contact. Also, Huang et al. (22) and Jia et al. (27) reported that Ali and other RAS inhibitors deteriorated the LV function after MI in animals and Solomon et al. (54) demonstrated that the use of RAS blockers did not attenuate the LV function significantly in high-risk patients at 36 weeks after MI. Also, Jia et al. (27) reported reduction in caspase-3 expression suggesting antiapoptotic effects for Ali. On the other hand, administration of Ali at high doses (50 and $100 \mathrm{mg} / \mathrm{kg}$ ) protects against the ISO-induced myocardial damage (30). 
This damaging effect for a low dose of Ali on ISO-induced myocardial injury could be explained by blocking of the cardiac non-circulatory RAS. In support with this hypothesis, in this study, we found no change in PRA for rats treated with both ISO and Ali, indicating that low dose of Ali did not attenuate the ISO-induced elevation in PRA. De Mello et al. (10) demonstrated that low dose of Ali ( 5 and $3 \mathrm{mg} / \mathrm{kg}$ ) significantly reduced the myocardial content of Ang II without affecting PRA suggesting that low dose of Ali mainly blocks cardiac not circulatory RAS. Also, Khan et al. (30) demonstrated that Ali at doses of 50 and $100 \mathrm{mg} / \mathrm{kg}$ blocked both the cardiac and the circulatory RAS completely. Moreover, previous studies demonstrated the upregulation of myocardial AT1R in ischemic MI $(34,44,64)$ and ISO-induced MI (31). These findings suggest that Ang II has more effects on the myocardium when we block RAS by small doses of Ali. Also, in this study, we can explain the damaging effects of a low dose of Ali on the myocardium by its hypotensive effect. The massive decrease of peripheral resistance and vasodilatation leads to diastolic and systolic pressure decrease, despite positive inotropic and chronotropic effects on the heart. In consistence with this hypothesis, in this study, we reported hypotension in the Ali groups at different times compared to ISO and NC groups. Also, Leenen et al. (33) demonstrated that the increased activity of the circulatory RAS appears to blunt the hypotensive effects of ISO. Also, it has been shown that ISO leads to the development of cardiac hypertrophy without increased blood pressure as a consequence of increased heart work $(28,43)$.

Moreover, in this study, the ISO-MI group showed significant increase in the duration of QRS complex, QT interval and ST segment elevation, and increase in HR. These ECG findings indicate the development of MI. These ECG findings of ISO-MI are in agreement with the previous studies $(58,63)$. Increased oxidative stress causes loss of cell membrane function leading to elevation of ST segment, conduction disturbances, and tachycardia (45). Furthermore, it has been demonstrated that an increase in HR is responsible for the increased oxygen consumption leading to accelerated myocardial necrosis (67). Also, the QT interval represents the period of electrical systole of the heart which is determined by inward $\mathrm{Na}^{+}$and $\mathrm{Ca}^{+2}$ currents and outward $\mathrm{K}^{+}$and $\mathrm{Cl}^{-}$currents (29) and is a method for determining the functional integrity of the myocardium (58). The QT interval prolongation may be related to cardiac vagal dysfunction and represents cardiac toxic potential, such as indication of arrhythmias, cardiac dysfunction, and sudden cardiac collapse (40). Changes in cardiac enzymes, cardiac morphology, and ECG suggest development of full picture of MI in ISO-induced toxicity. Interestingly, Ali did not cause any improvement in these ECG variables. Moreover, Ali caused significant increase in HR and reduction in duration of T-wave and makes it tall and peaked, which may be a reflection of hyperkalemia induced by Ali treatment.

Mitochondria represent major sites for generation of ROS as a by-product of oxidative phosphorylation. Mitochondrial proteins, lipids, and DNA are believed to be primary targets of oxidative damage during stress (1), thus creating a mitochondrial free radical "vicious cycle" of injury. Dai et al. (7) provided direct evidence that amplification of ROS within mitochondria is a key mediator of Ang II-induced cardiac hypertrophy and failure. Several studies have postulated that Ang II may promote the ROS generation in mitochondria through the ROS-induced ROS release mechanism $(3,9,69)$. When, Ang II binds to Ang II type 1 receptor, it activates NADPH oxidase through a protein kinase $\mathrm{C}$ (PKC)-dependent manner to increase ROS production (13). This $\mathrm{NAD}(\mathrm{P}) \mathrm{H}$ oxidase-derived ROS may serve as a trigger to induce mitoK $\mathrm{ATP}_{\mathrm{AT}}$ channel opening and mitochondrial permeability transition pore formation, depolarize mitochondrial membrane potential $\left(\Delta \psi_{\mathrm{M}}\right)$, and lead to the mitochondrial ROS 
burst (68). Previous studies demonstrated that mice overexpressing CAT targeted to mitochondria have shown to be resistant to cardiac hypertrophy, fibrosis, and mitochondrial damage induced by Ang II. Therefore, mitochondria-targeted antioxidants have been suggested as promising agents for prevention and treatment of hypertensive cardiomyopathy $(7,8)$.

The oxidative stress plays an important role in the detrimental effects caused by ISO. In this study, we demonstrated significant elevation in MDA levels in myocardium, which was associated with significant reduction in the anti-oxidant activity of CAT and GSH concentrations in the heart tissues. Lipid peroxidation (LPO) product MDA is the result of oxidation of polyunsaturated fatty acids in the cell membranes, which may cause a decrease in membrane fluidity and its disruption and impairment of its function (49). The reduction in the antioxidants, GSH and CAT, could be explained by their excessive utilization during the burst of ROS production, in protecting SH group containing proteins from LPO (20). These antioxidants limit the toxicity associated with free radicals and expected to be consumed by enhanced radical reactions (57). Also, the present results show that low dose of Ali treatment caused a significant increase in MDA levels suggesting the oxidant effects of Ali and explained the damaging effects of Ali on myocardium partially on basis of enhanced oxidative stress. We hypothesized that upregulation of AT1R and elevated circulatory RAS (by low dose of Ali) in ISO-MI increases the production of ROS through NADPH oxidasedependent pathway $(2,4,5)$ which is activated by AT1R. In support of this hypothesis, Khan et al. (30) found that 50 and $100 \mathrm{mg} / \mathrm{kg}$ doses cause significant attenuation of the enhanced expression of caspase-3 and oxidative stress with more pronounced effect for $100 \mathrm{mg} / \mathrm{kg}$.

In this study, we investigated the expression of Nrf2/HO-1 pathways in ISO-MI and the impact of renin inhibition on this pathway. Santos et al. (51) reported that a high dose of ISO can induce ROS production, which activates diverse signaling pathways such as redoxsensitive transcription factors. Nrf2 is a redox-sensitive transcription factor, which regulates the induction of phase 2 detoxifying and antioxidant genes (56). Li et al. $(36,37)$ demonstrated that $\mathrm{Nrf} 2$ is a negative regulator of oxidative stress in cardiomyocytes and observed that Ang II-activated Nrf2 in the heart and Nrf2 deficiency exaggerated Ang IIinduced cardiac hypertrophy. They concluded that increased Nrf2 activity in the myocardium is secondary to the Ang II-induced oxidative stress because a robust increase in ROS formation appeared prior to Nrf2 activation in Ang II-stimulated cardiomyocytes in vitro. Additionally, Nrf2 overexpression suppressed basal ROS formation and blocked Ang IIinduced ROS production in cultured cardiomyocytes, whereas Nrf2 knockdown dramatically enhanced the basal ROS formation without further increases in Ang II-stimulated ROS production. Nguyen et al. (42) demonstrated that overexpression of Nrf2 eliminates reactive oxidants, whereas impaired Nrf2 activity showed the opposite effects. In this study, the ISO-MI group showed a significant increase in Nrf2 expression due to the presence of ROS, which in turn activates Nrf2. In consistence with this hypothesis, previous studies demonstrated that mitochondria-derived ROS induce the activation of $\operatorname{Nrf2}(35,53)$. Also, Li et al. (35) demonstrated a significant increase in Keap1 expression in ISO-treated rats, which indicates that Nrf2 was rather associated with Keap1, and thus with less translocation of Nrf2 in the nucleus to activate antioxidant gene expression. Under oxidative stress or other potentially damaging stimuli, Nrf2 dissociates from Keap 1 and translocates into the nucleus, where it binds to the antioxidant response element sequences, leading to the transcriptional activation of phase II enzymes/antioxidant genes, including HO-1, NQO1, GST, GCL, etc. In 
this study, we demonstrated a significant increase in Nrf2 in ISO-MI group compared to NC group suggesting enhanced expression of Nrf2 in ISO to overcome the oxidative stress in ISO-MI. Nrf2-mediated antioxidant and phase II enzymes may contribute to cellular protection against oxidative stress and to potentiate antioxidant defense capacity in cells. Unfortunately, we found that low dose of Ali caused significant attenuation in $\mathrm{Nrf} 2$ expression suggesting attenuation of $\mathrm{Nrf} 2$ (endogenous protective mechanism against oxidative stress damage) by excessive ROS, which enhance the myocardial damage as laboratory and histopathogical examinations reported.

HO-1 is a cytoprotective enzyme that is induced by stress and participates in the homeostatic control of cardiovascular functions, including the regulation of blood pressure and the prevention of cardiac ischemic injury (60). Previous studies demonstrated acute HO-1 upregulation in response to ischemic injury in several organs, including the heart $(17,32,39$, 66). Also, previous studies demonstrated that pharmacological activation of HO-1 ameliorates post-infarction myocardial remodeling (6) and hepatic ischemic injury (15) and modulate renal hemodynamics and renal excretory function in renal ischemia (16). In this study, we found that ISO caused a significant increase in the expression of HO-1 and treatment with Ali at low dose caused minimal reduction in HO-1 suggesting that DRI at low dose makes more stress on myocardial cells.

Also, in this study, we demonstrated increased apoptosis in the myocardium in the form of low expression of Bcl2. Although Ali caused significant deterioration in myocardial functions and morphology, it caused a significant increase in the anti-apoptotic protein $\mathrm{Bcl} 2$. In consistence with these findings, Jia et al. (27) reported that Ali ameliorated the cardiomyocytic apoptosis (caspase-3), attenuated the sympathetic nerve innervations (tyrosine hydroxylase, an GAP43 protein expression) and sprouting and reduced the vulnerability of ventricular arrhythmias after ischemic MI. Also, Khan et al. (30) demonstrated that Ali at high dose $(50$ and $100 \mathrm{mg} / \mathrm{kg})$ significantly attenuated the significant increase in caspase-3 expression induced by ISO. Enalapril $(10 \mathrm{mg} / \mathrm{kg} / \mathrm{day})$, valsartan (10 $\mathrm{mg} / \mathrm{kg} /$ day), and carvedilol (beta adrenergic blocker $20 \mathrm{mg} / \mathrm{kg} / \mathrm{day}$ ) (by the same regime of Ali) have similar effects as Ali on cardiomyocytic apoptosis, sympathetic nerve innervations, and vulnerability of ventricular arrhythmias after MI. Westermann et al. (61) showed that Ali treatment for 10 days in ischemic MI (10 and $50 \mathrm{mg} / \mathrm{kg})$ did not affect systemic blood pressure and improved systolic and diastolic LV function, LV dilatation, cardiac hypertrophy, and lung weights. This was associated with a normalization of the mitogen-activated protein kinase P38 and extracellular signal-regulated kinases $1 / 2$, AKT, and the apoptotic markers Bax and Bcl2 (all measured by Western blots), as well as the number of terminal deoxynucleotidyl transferase dUTP nick end labeling-positive cells in histology. LV dilatation, as well as the associated upregulation of gene expression (mRNA abundance) and activity (by zymography) of the cardiac metalloproteinase 9 in the placebo group after MI, was also attenuated in the Ali-treated group.

We concluded that early inhibition of RAS by Ali at low dose in ISO-MI enhanced the ISOinduced deteriorations in myocardial functions and morphology caused by ISO in rats. This might be due to enhanced oxidative stress and apoptosis and downregulation of Nrf2 and HO-1.

\section{Acknowledgements}

The authors would like to thank Dr. Azza Abdelaziz, Assistant professor of pathology for helping us in histopathological examination. They would also like to express their gratitude to King Khalid University, Abha, Saudi Arabia and Mansoura University, Mansoura, Egypt for providing administrative and technical support. 


\section{REFERENCES}

1. Balaban RS, Nemoto S, Finkel T: Mitochondria, oxidants, and aging. Cell 120, $483-495$ (2005)

2. Bendall JK, Cave AC, Heymes C, Gall N, Shah AM: Pivotal role of a gp91(phox)-containing NADPH oxidase in angiotensin II-induced cardiac hypertrophy in mice. Circulation 105, 293-296 (2002)

3. Brady NR, Hamacher-Brady A, Westerhoff HV, Gottlieb RA: A wave of reactive oxygen species (ROS)-induced ROS release in a sea of excitable mitochondria. Antioxid. Redox Signal. 8, 1651-1665 (2006)

4. Brown JH, Del Re DP, Sussman MA: The Rac and Rho hall of fame: a decade of hypertrophic signaling hits. Circ. Res. 98, 730-742 (2006)

5. Byrne JA, Grieve DJ, Bendall JK, Li JM, Gove C, Lambeth JD, Cave AC, Shah AM: Contrasting roles of NADPH oxidase isoforms in pressure-overload versus angiotensin II-induced cardiac hypertrophy. Circ. Res. 93, 802-805 (2003)

6. Collino M, Pini A, Mugelli N, Mastroianni R, Bani D, Fantozzi R, Papucci L, Fazi M, Masini E: Beneficial effect of prolonged heme oxygenase-1 activation in a rat model of chronic heart failure. Dis Model Mech. 6(4), $1012-1020$ (2013)

7. Dai DF, Chen T, Szeto H, Nieves-Cintrón M, Kutyavin V, Santana LF, Rabinovitch PS: Mitochondrial targeted antioxidant peptide ameliorates hypertensive cardiomyopathy. J. Am. Coll. Cardiol. 58, 73-82 (2011)

8. Dai DF, Johnson SC, Villarin JJ, Chin MT, Nieves-Cintrón M, Chen T, Marcinek DJ, Dorn GW, Kang YJ, Prolla TA: Mitochondrial oxidative stress mediates angiotensin II-induced cardiac hypertrophy and Galphaq overexpression-induced heart failure. Circ. Res. 108, 837-846 (2011)

9. Daiber A: Redox signaling (cross-talk) from and to mitochondria involves mitochondrial pores and reactive oxygen species. Biochim. Biophys. Acta 1797, 897-906 (2010)

10. De Mello W, Rivera M, Rabell A, Gerena Y: Aliskiren, at low doses, reduces the electrical remodeling in the heart of the TGR(mRen2)27 rat independently of blood pressure. J. Renin Angiotensin Aldosterone Syst. 4, 23-33 (2013)

11. Devika PT, Stanely Mainzen Prince P: Protective effect of (-)-epigallocatechingallate (EGCG) on lipid peroxide metabolism in ISO induced myocardial infarction in male Wistar rats: a histopathological study. Biomed. Pharmacother. 62, 701-708 (2008)

12. Dhalla N, Yates J, Naimark B (1992): Cardio toxicity of catecholamines and related agents. In: Cardiovascular Toxicology (2nd ed.), ed Acosta D, Raven Press, New York, pp. 239-282

13. Doughan AK, Harrison DG, Dikalov SI: Molecular mechanisms of angiotensin II-mediated mitochondrial dysfunction: linking mitochondrial oxidative damage and vascular endothelial dysfunction. Circ. Res. 102, 488-496 (2008)

14. Duprez DA, Munger MA, Botha J, Keefe DL, Charney AN: Aliskiren for geriatric lowering of systolic hypertension: a randomized controlled trial. J. Hum. Hypertens. 24, 600-608 (2010)

15. Fang J, Qin H, Seki T, Nakamura H, Tsukigawa K, Shin T, Maeda H: Therapeutic potential of pegylated hemin for reactive oxygen species-related diseases via induction of heme oxygenase-1: results from a rat hepatic ischemia/reperfusion injury model. J. Pharmacol. Exp. Ther. 339, 779-789 (2011)

16. Ferenbach DA, Kluth DC, Hughes J: Heme oxygenase-1 and renal ischaemia-reperfusion injury. Nephron. Exp. Nephrol. 115, e33-e37 (2010)

17. Giannini L, Vannacci A, Fabrizi F, Uliva C, Bani D, Masini E, Mannaioni PF: Protection from cardiac injury by induction of heme oxygenase- 1 and nitric oxide synthase in a focal ischaemia-reperfusion model. Cell. Mol. Biol. (Noisy-le-grand) 51, 393-401 (2005)

18. Gullapalli N, Bloch M, Basile J: Renin-angiotensin-aldosterone system blockade in high-risk hypertensive patients: current approaches and future trends. Ther. Adv. Cardiovasc. Dis. 4, 359-373 (2010)

19. Guo BY, Li YJ, Han R, Yang SL, Shi YH, Han DR, Zhou H, Wang M: Telmisartan attenuates isoproterenolinduced cardiac remodeling in rats via regulation of cardiac adiponectin expression. Acta Pharmacol. Sin. 32, $449-455$ (2011)

20. Halliwell B, Gutteridge JM: Role of free radicals and catalytic metal ions in human disease: an overview. Methods Enzymol. 186, 1-85 (1990)

21. Higashikuni Y, Takaoka M, Iwata H, Tanaka K, Hirata Y, Nagai R, Sata M: Aliskiren in combination with valsartan exerts synergistic protective effects against ventricular remodeling after myocardial infarction in mice. Hypertens. Res. 35, 62-69 (2012)

22. Huang BS, Ahmad M, Tan J, Leenen FH: Chronic central versus systemic blockade of AT(1) receptors and cardiac dysfunction in rats post myocardial infarction. Am. J. Physiol. Heart Circ. Physiol. 297, H968-H975 (2009)

23. Hussein Ael-A, Abbas AM, El Wakil GA, Elsamanoudy AZ, El Aziz AA: Effect of chronic excess iodine intake on thyroid function and oxidative stress in hypothyroid rats. Can. J. Physiol. Pharmacol. 90(5), 617-625 (2012) 
24. Issan Y, Kornowski R, Aravot D, Shainberg A, Laniado-Schwartzman M, Sodhi K, Abraham NG, Hochhauser E: Heme oxygenase-1 induction improves cardiac function following myocardial ischemia by reducing oxidative stress. PLoS One 9(3), e92246 (2014)

25. Iusuf D, Henning RH, van Gilst WH, Roks AJ: Angiotensin-(1-7): pharmacological properties and pharmacotherapeutic perspectives. Eur. J. Pharmacol. 585(2-3), 303-312 (2008)

26. Jennings RB, Reimer KA: The cell biology of acute myocardial ischemia. Annu. Rev. Med. 42, 225-246 (1991)

27. Jia YY, Bao ZW, Wei MF, Zhu JH, Gui L: Aliskiren ameliorates sympathetic nerve sprouting and suppresses the inducibility of ventricular tachyarrhythmia in postinfarcted rat heart. Chin. Med. J. (Engl). 126, 4707-4714 (2013)

28. Kaye D, Esler M: Sympathetic neuronal regulation of the heart in aging and heart failure. Cardiovasc. Res. 66, 256-264 (2005)

29. Kela AK, Reddy LP, Thombre DP: E.C.G. findings in normal rats and after administration of isoproterenol. Ind. J. Physiol. Pharmacol. 24, 84-90 (1980)

30. Khan V, Hassan MQ, Akhtar M, Najmi AK: Renin inhibition by aliskiren protects rats against isoproterenol induced myocardial infarction. Drug Res. (Stuttg). 68, 139-145 (2018)

31. Kuipers I, van der Harst P, Kuipers F, van Genne L, Goris M, Lehtonen JY, van Veldhuisen DJ, van Gilst WH, de Boer RA: Activation of liver X receptor-alpha reduces activation of the renal and cardiac renin-angiotensinaldosterone system. Lab. Invest. 90, 630-636 (2010)

32. Lakkisto P, Csonka C, Fodor G, Bencsik P, Voipio-Pulkki LM, Ferdinandy P, Pulkki K: The heme oxygenase inducer hemin protects against cardiac dysfunction and ventricular fibrillation in ischaemic/reperfused rat hearts: role of connexin 43. Scand. J. Clin. Lab. Invest. 69, 209-218 (2009)

33. Leenen FH, White R, Yuan B: Isoproterenol-induced cardiac hypertrophy: role of circulatory versus cardiac renin-angiotensin system. Am. J. Physiol. Heart Circ. Physiol. 281, H2410-H2416 (2001)

34. Li C, Han R, Kang L, Wang J, Gao Y, Li Y, He J, Tian J: Pirfenidone controls the feedback loop of the AT1R/ p38 MAPK/renin-angiotensin system axis by regulating liver $\mathrm{X}$ receptor- $\alpha$ in myocardial infarction-induced cardiac fibrosis. Sci. Rep. 7, 40523 (2017)

35. Li H, Song F, Duan LR, Sheng JJ, Xie YH, Yang Q, Chen Y, Dong QQ, Zhang BL, Wang SW: Paeonol and danshensu combination attenuates apoptosis in myocardial infarcted rats by inhibiting oxidative stress: roles of Nrf2/HO-1 and PI3K/Akt pathway. Sci. Rep. 6, 23693 (2016)

36. Li J, Ichikawa T, Villacorta L, Janicki JS, Brower GL, Yamamoto M, Cui T: Nrf2 protects against maladaptive cardiac responses to hemodynamic stress. Arterioscler. Thromb. Vasc. Biol. 29, 1843-1850 (2009)

37. Li J, Zhang C, Xing Y, Janicki JS, Yamamoto M, Wang XL, Tang DQ, Cui T: Up-regulation of p27 (kip1) contributes to Nrf2-mediated protection against angiotensin II-induced cardiac hypertrophy. Cardiovasc. Res. 90, 315-324 (2011)

38. Lopez AD, Murrau CC: The global burden disease, 1990-2020. Nat. Med. 4, 1241-1243 (1998)

39. Masini E, Vannacci A, Marzocca C, Pierpaoli S, Giannini L, Fantappie O, Mazzanti R, Mannaioni PF: Heme oxygenase-1 and the ischemia reperfusion injury in the rat heart. Exp. Biol. Med. (Maywood) 228, 546-549 (2003)

40. Mazzoleni A, Cutin ME, Wolff R, Reiner L, Somes G: On the relationship between heart weights, fibrosis, and QRS duration. J. Electrocardiol. 8, 233-236 (1975)

41. Metias EF, Aboelmaaty NM, Hussein AM, Abdallah EW, Abdelaziz A: Modulation of ECG, myocardial oxidative stress markers and connexion 43 expression by ascorbic acid and ferulic acid in isoproterenol-induced myocardial infarction in rats. Biochem. Physiol. 5, 210 (2016)

42. Nguyen T, Nioi P, Pickett CB: The Nrf2-antioxidant response element signaling pathway and its activation by oxidative stress. J. Biol. Chem. 284, 13291-13295 (2009)

43. Ozaki M, Kawashima S, Yamashita T, Hirase T, Ohashi Y, Inoue N, Hirata K, Yokoyoma M: Overexpression of endothelial nitric oxide synthase attenuates cardiac hypertrophy induced by chronic isoproterenol infusion. Circulation 66, 851-856 (2002)

44. Parodi-Rullan R, Barreto-Torres G, Ruiz L, Casasnovas J, Javadov S: Direct renin inhibition exerts an antihypertrophic effect associated with improved mitochondrial function in postinfarction heart failure in diabetic rats. Cell. Physiol. Biochem. 29, 841-850 (2012)

45. Prince SMP, Rajkumar S, Dhanasekar K: Protective effects of vanillic acid on electrocardiogram, lipid peroxidation, antioxidants, proinflammatory markers and histopathology in isoproterenol induced cardiotoxic rats. Eur. J. Pharmacol. 668, 233-240 (2011)

46. Rajadurai M, Prince SMP: Preventive effect of naringin on lipid peroxides and antioxidants in isoproterenolinduced cardiotoxicity in Wistar rats: biochemical and histopathological evidences. Toxicology 228, 259-268 (2006) 
47. Ramirez TA, Iyer RP, Ghasemi O, Lopez EF, Levin DB, Zhang J, Zamilpa R, Chou YM, Jin YF, Lindsey ML: Aliskiren and valsartan mediate left ventricular remodeling post-myocardial infarction in mice through MMP-9 effects. J. Mol. Cell. Cardiol. 72, 326-335 (2014)

48. Rona G: Catecholamine cardiotoxicity. J. Mol. Cell. Cardiol. 17, 291-306 (1985)

49. Rona G, Zsoter T, Chappel C, Gaudry R: Myocardial lesions, circulatory and electrocardiographic changes produced by isoproterenol in the dog. Rev. Can. Biol. 18, 83-94 (1959)

50. Sahu BD, Anubolu H, Koneru M, Kumar JM, Kuncha M, Rachamalla SS, Sistla R: Cardioprotective effect of embelin on isoproterenol-induced myocardial injury in rats: possible involvement of mitochondrial dysfunction and apoptosis. Life Sci. 107, 59-67 (2014)

51. Santos CXC, Anilkumar N, Zhang M, Brewer AC, Shah AM: Redox signaling in cardiac myocytes. Free Radic. Biol. Med. 50, 777-793 (2011)

52. Sathish V, Ebenezar KK, Devaki T: Synergistic effect of nicorandil and amlodipine on tissue defense system during experimental myocardial infarction in rats. Mol. Cell. Biochem. 243, 133-138 (2003)

53. Shih AY, Imbeault S, Barakauskas V, Erb H, Jiang L, Li P, Murphy TH: Induction of the Nrf2-driven antioxidant response confers neuroprotection during mitochondrial stress in vivo. J. Biol. Chem. 280, 22925-22936 (2005)

54. Solomon SD, Shin SH, Shah A, Skali H, Desai A, Kober L, Maggioni AP, Rouleau JL, Kelly RY, Hester A, McMurray JJ, Pfeffer MA, Aliskiren Study in Post-MI Patients to Reduce Remodeling (ASPIRE) Investigators: Effect of the direct renin inhibitor aliskiren on left ventricular remodelling following myocardial infarction with systolic dysfunction. Eur. Heart J. 32, 1227-1234 (2011)

55. Staessen JA, Li Y, Richart T: Oral rennin inhibitors. Lancet 368, 1449-1456 (2006)

56. $\mathrm{Su}$ JD, Yen JH, Li S, Weng CY, Lin MH, Ho CT, Wu MJ: 3',4'-Didemethylnobiletin induces phase II detoxification gene expression and modulates PI3K/Akt signaling in PC12 cells. Free Radic. Biol. Med. 52, 126-141 (2012)

57. Sun F, Hamagawa E, Tsutsui C, Sakaguchi N, Kakuta Y, Tokumaru S, Kojo S: Evaluation of oxidative stress during apoptosis and necrosis caused by D-galactosamine in rat liver. Biochem. Pharmacol. 65, 101-107 (2003)

58. Thippeswamy BS, Thakker SP, Tubachi S, Kalyani GA, Netra MK, Patil U, Desai S, Gavimath CC, Veerapur VP: Cardioprotective effect of Cucumis trigonus Roxb on isoproterenol-induced myocardial infarction in rat. Am. J. Pharmacol. Toxicol. 4, 29-37 (2009)

59. Upaganlawar A, Gandhi H, Balaraman R: Isoproterenol induced myocardial infarction: protective role of natural products. J. Pharmacol. Toxicol. 6, 1-17 (2011)

60. Wang G, Hamid T, Keith RJ, Zhou G, Partridge CR, Xiang X, Kingery JR, Lewis RK, Li Q, Rokosh DG, Ford R, Spinale FG, Riggs DW, Srivastava S, Bhatnagar A, Bolli R, Prabhu SD: Cardioprotective and antiapoptotic effects of heme oxygenase-1 in the failing heart. Circulation 121, 1912-1925 (2010)

61. Westermann D, Riad A, Lettau O, Roks A, Savvatis K, Becher PM, Escher F, Jan Danser AH, Schultheiss HP, Tschöpe C: Renin inhibition improves cardiac function and remodeling after myocardial infarction independent of blood pressure. Hypertension 52, 1068-1075 (2008)

62. Xia HJ, Dai DZ, Dai Y: Up-regulated inflammatory factors endothelin, NFkB, TNFalpha. and iNOS involed in exaggerated cardiac arrhythmias in L-thyroxine- induced cardiomyopathy are suppressed by darusentan in rats. Life Sci. 79, 1812-1819 (2006)

63. Yadav $\mathrm{CH}$, Akhtar M, Khanam R: Isoproterenol toxicity induced ECG alterations in Wistar rats: role of histamine H3 receptor agonist imetit. Int. J. Pharm. Pharm. Sci. 6, 654-658 (2014)

64. Yang BC, Phillips MI, Ambuehl PEJ, Shen LP, Mehta P, Mehta JL: Increase in angiotensin II type 1 receptor expression immediately following ischemia-reperfusion in isolated rat hearts. Circulation 96, 922-926 (1997)

65. Yang N-I, Liao C-C, Hung M-J, Cherng W-J: Direct renin inhibitor attenuates left ventricular remodeling in post-myocardial infarction heart failure mice. Acta Cardiol. Sin. 29, 160-167 (2013)

66. Yeh $\mathrm{CH}$, Chen TP, Wang YC, Lin YM, Lin PJ: HO-1 activation can attenuate cardiomyocytic apoptosis via inhibition of NF-kappaB and AP-1 translocation following cardiac global ischemia and reperfusion. J. Surg. Res. $155,147-156$ (2009)

67. Yogeeta SK, Gnanapragasam A, Senthil Kumar S, Subashini R, Devaki T: Synergistic interactions of ferulic acid with ascorbic acid: its cardioprotective role during isoproterenol induced myocardial infarction in rats. Mol. Cell. Biochem. 283, 139-146 (2006)

68. Zhang GX, Lu XM, Kimura S, Nishiyama A: Role of mitochondria in angiotensin II-induced reactive oxygen species and mitogen-activated protein kinase activation. Cardiovasc. Res. 76, 204-212 (2007)

69. Zorov DB, Juhaszova M, Sollott SJ: Mitochondrial ROS-induced ROS release: an update and review. Biochim. Biophys. Acta 1757, 509-517 (2006) 